\title{
MEMS-based extreme adaptive optics for planet detection
}

B. A. Macintosh, J. R. Graham, B. Oppenheimer, L. Poyneer, A. Sivaramakrishnan, J.P. Veran

November 21, 2005

SPIE CONFERENCE 6113: MEMS/MOEMS COMPONENTS AND THEIR APPLICATIONS III

San Jose, CA, United States

January 21, 2006 through January 26, 2006 
This document was prepared as an account of work sponsored by an agency of the United States Government. Neither the United States Government nor the University of California nor any of their employees, makes any warranty, express or implied, or assumes any legal liability or responsibility for the accuracy, completeness, or usefulness of any information, apparatus, product, or process disclosed, or represents that its use would not infringe privately owned rights. Reference herein to any specific commercial product, process, or service by trade name, trademark, manufacturer, or otherwise, does not necessarily constitute or imply its endorsement, recommendation, or favoring by the United States Government or the University of California. The views and opinions of authors expressed herein do not necessarily state or reflect those of the United States Government or the University of California, and shall not be used for advertising or product endorsement purposes. 


\title{
MEMS-based extreme adaptive optics for planet detection
}

\author{
Bruce Macintosh $^{* a b}$, James Graham ${ }^{\text {ae }}$, Ben Oppenheimer ${ }^{\text {ad }}$, Lisa Poyneer ${ }^{\text {ab }}$, Anand \\ Sivaramakrishnan $^{\text {ad }}$, and Jean-Pierre Veran ${ }^{\mathrm{f}}$ \\ ${ }^{a}$ NSF Center for Adaptive Optics \\ ${ }^{\mathrm{b}}$ Lawrence Livermore National Laboratory, 7000 East Ave., Livermore, CA 94551 \\ ${ }^{\mathrm{c}}$ Space Telescope Science Institute, 3700 San Martin Drive, Baltimore, MD 21218 \\ ${ }^{\mathrm{d}}$ Astrophysics Department, The American Museum of Natural History, $79^{\text {th }}$ Street at Central Park \\ West, New York, NY 10024-5192 \\ ${ }^{\mathrm{e}}$ Department of Astronomy, University of California at Berkeley, Berkeley, CA 94720 \\ ${ }^{\mathrm{f}}$ Herzberg Institute of Astrophysics, 5071 W. Saanich Road, Victoria, Canada
}

\begin{abstract}
The next major step in the study of extrasolar planets will be the direct detection, resolved from their parent star, of a significant sample of Jupiter-like extrasolar giant planets. Such detection will open up new parts of the extrasolar planet distribution and allow spectroscopic characterization of the planets themselves. Detecting Jovian planets at 5-50 AU scale orbiting nearby stars requires adaptive optics systems and coronagraphs an order of magnitude more powerful than those available today - the realm of "Extreme" adaptive optics. We present the basic requirements and design for such a system, the Gemini Planet Imager (GPI.) GPI will require a MEMS-based deformable mirror with good surface quality, 2-4 micron stroke (operated in tandem with a conventional low-order "woofer" mirror), and a fully-functional 48actuator-diameter aperture.
\end{abstract}

Keywords: Adaptive optics, extrasolar planets, speckle, coronagraphs

\section{INTRODUCTION AND SCIENTIFIC MOTIVATION}

One of the most active areas in astronomy in recent years has been the study of extrasolar planets. Since 2005 over 160 planets have been discovered orbiting stars other than the sun. ${ }^{1}$ The vast majority have been detected through measurements of the doppler shift of the parent star caused by the gravitational influence of the planet. Figure 1 shows the distribution of these planets in a mass/orbital semi-major axis space. Most have masses greater than Jupiter, and are almost all are within 5 astronomical units (AU) of their parent star - a very different distribution than the giant planets of our solar system. The very existence of these planets was a considerable surprise to most astronomers. Conventional models of planet formation predicted that giant planets could only form in the outer parts of systems. Young stars are surrounded by a circumstellar disk of gas, ice, and dust. In the conventional model, beyond the $\sim 3$ AU "snow line" water ice particles would assemble through collisions into planetesimals and ultimately into a planetary core that would then rapidly accrete gas from the disk, forming a giant gaseous planet such as Jupiter or Saturn. Inside the snow line, only (rarer) rocky materials are solid, requiring a much larger time for the formation of planetary cores; by the time a core has reached a substantial mass, no gas remains to be accreted, and the core becomes a solid planet such as Venus or the Earth.

\footnotetext{
*bmac@igpp.llnl.gov
} 


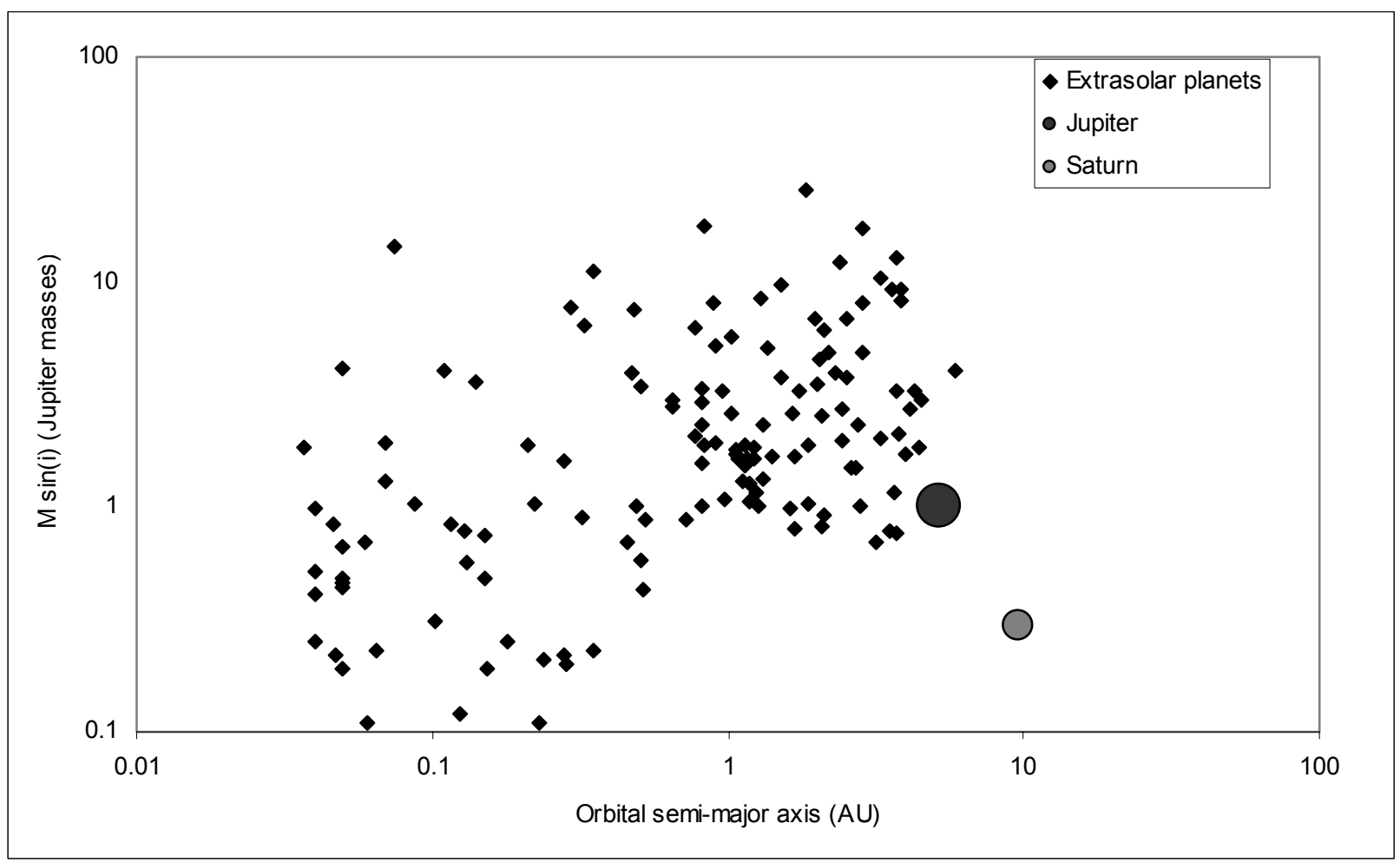

Figure 1: Known extrasolar planets as of February $2005^{1}$, plotted in mass (multiplied by the sine of the unknown inclination) times semi-major axis space. (For circular orbits, the semi-major axis is the orbital radius.)

Now that we know of the existence of giant planets close to their parent stars, this paradigm has been modified. The most common explanation is that giant planets still form at $>3 \mathrm{AU}$ but then migrate inward, either due to tidal friction with the disk ${ }^{2}$ or through gravitational interactions between multiple planets in the same system ${ }^{3}$. A third possibility is that planets form not through gradual accretion but through rapid fragmentation of a circumstellar disk ${ }^{4}$. These models have different implications for the existence of Earthlike planets and solar systems resembling our own, but current statistics and observations are insufficient to distinguish between these scenarios. Each makes different predictions for the properties of planets with orbits $>5 \mathrm{AU}$.

Figure 1 shows almost no planets beyond $5 \mathrm{AU}$, but this does not mean such planets are rare. The Doppler technique is preferentially sensitive to planets close to their star. First, a planet must be massive enough and close enough to its parent star to induce a detectable Doppler shift. Second, and more fundamental, to measure an orbit the planet must complete at least one circuit around its parent. Orbital period $P$ in years is given by Kepler's third law:

$$
P^{2}=\frac{A^{3}}{M_{*}}
$$

where $A$ is the orbital semi-major axis in AU and $M_{*}$ is the mass of the parent star in units of the mass of our sun. Jupiter in our solar system has a 11.9 years. High-accuracy Doppler searches of large numbers of stars, e.g. on the Keck telescope ${ }^{5}$ have only been in process since 1996; the absence of Jupiter analogs in Figure 1 is due solely to the fact that insufficient time has passed to detect them. To detect a planet like Saturn will require 29 years; Neptune, 164 years. The ability of Doppler techniques to probe outer solar systems is therefore limited by the patience and lifetime of astronomers.

A powerful complement to Doppler techniques would be the direct imaging detection of photons from the extrasolar planets themselves. Imaging detection is primarily sensitive to planets in wide orbits, where scattered starlight is less of a problem. Imaging techniques require only a short period of time rather than a full orbit, and (through photometry or 
spectroscopy) can measure the properties of the planet itself, such as radius, temperature, and composition. Unfortunately such detection is extremely challenging. Jupiter, seen from outside our solar system, is $10^{9}$ times fainter than the sun; a Jupiter analog in another solar system would be swamped by the light from its parent star, scattered by diffraction, optical imperfections, and atmospheric turbulence. At infrared wavelengths, younger and more massive planets will be brighter( Figure 2), and a small number of candidate planets have been detected using current adaptive optics in exotic circumstances ${ }^{6}$. However, to truly detect a significant sample of extrasolar planets requires achieving contrasts of $10^{7}-10^{8}$.

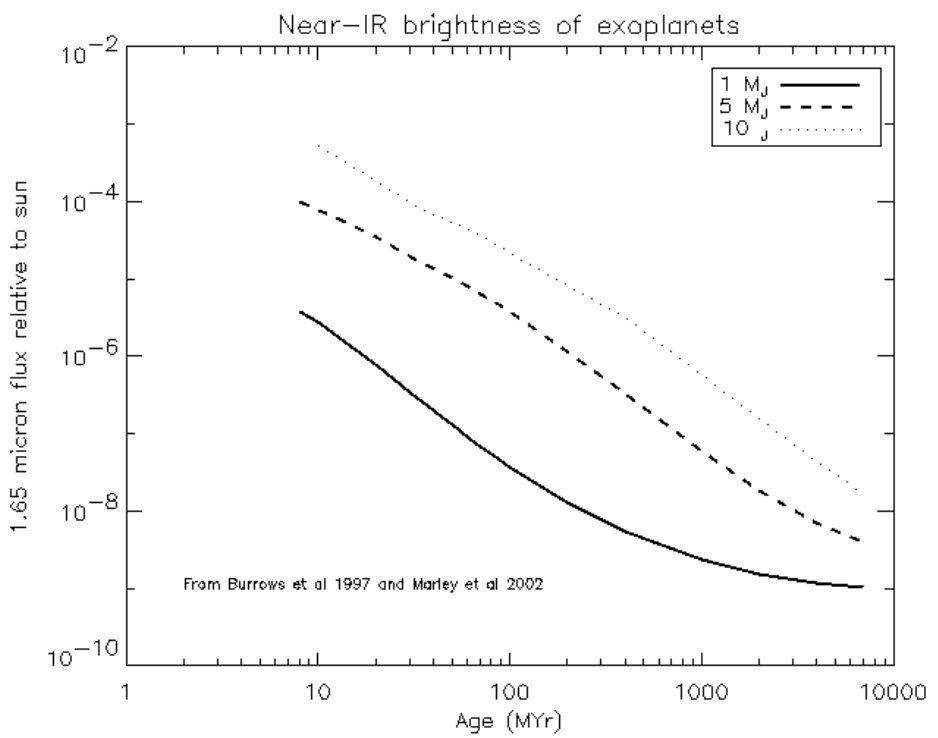

Figure 2: Near-infrared brightness of extrasolar planets ${ }^{7}$, expressed as the ratio of the planet's luminosity to that of a solar-type star, integrated over a wavelength of 1.5-1.8 $\mu \mathrm{m}$.

Current adaptive optics (AO) systems are insufficient to achieve this. In the past several years, adaptive optics systems have come into routine use on the world's largest telescopes. These systems have been designed for general purpose use, balancing the desire for good final wavefront quality with the flux available for wavefront measurement from 5-20 $\mathrm{W}$ artificial sodium-layer laser beacons ${ }^{8}$ or from a large enough sample of natural stars, which will necessarily be relatively $\operatorname{dim}\left(\sim 14^{\text {th }}\right.$ magnitude at 550 nanometers, corresponding to a flux of $\sim 0.025$ photons $\left.\mathrm{cm}^{-2} \mathrm{~second}^{-1} \mathrm{~nm}^{-1}\right)$. This balancing leads to systems whose deformable mirrors map to subaperture sizes $d=50-80 \mathrm{~cm}$ on the telescope primary mirror, with total RMS wavefront errors in the 200-400 nm range and Strehl ratio $S$ of $0.2-0.7$ at a wavelength of 2.1 $\mu \mathrm{m}$. At these Strehl ratios, a star is still surrounded by a broad halo of scattered light containing $1-S=30-80 \%$ of the star's light. With long exposures and careful image processing, contrasts of $10^{5}-10^{6}$ can be achieved at relatively wide separations ( $>1$ arcsecond.) Since a separation of 1 AU subtends an angle of one arcsecond at a distance of 1 parsec, and a typical $10 \mathrm{Myr}$ young star with still-detectable planets will be $\sim 50$ parsecs away, this allows detection of only very young planets in very wide ( $>50 \mathrm{AU})$ orbits. To truly detect extrasolar planets will require the next generation of socalled "Extreme" adaptive optics (ExAO) systems, with dense actuator spacing ( $<25 \mathrm{~cm})$, Strehl ratios approaching unity, careful control of static wavefront errors, and a coronagraph to block diffraction.

\section{POINT SPREAD FUNCTION OF A HIGH-STREHL AO SYSTEM}

We express the electric field in the pupil plane of an AO-corrected telescope as $E(\mathbf{x})=A(\mathbf{x}) e^{i \phi(\mathbf{x})}$ where $\mathbf{x}=(x, y)$ is a coordinate vector in the pupil plane. The resulting point spread function (PSF) can be expressed as a Taylor series expansion of the Fourier transform of this quantity squared; in the high Strehl ratio regime this reduces to 910 


$$
\begin{aligned}
p(\mathbf{k}) \cong & a a^{*} \\
& -i\left[a\left(a^{*} * \Phi^{*}\right)-a^{*}(a * \Phi)\right] \\
& +(a * \Phi)\left(a^{*} * \Phi^{*}\right)-\frac{1}{2}\left[a\left(a^{*} * \Phi^{*} * \Phi^{*}\right)+a^{*}(a * \Phi * \Phi)\right]+\ldots
\end{aligned}
$$

where $a$ and $\Phi$ are the Fourier transforms of $A$ and $\phi$ respectively, * denotes convolution, and $\mathbf{k}=2 \pi\left(\theta_{\mathrm{x}}, \theta_{\mathrm{y}}\right) / \lambda$ is the spatial frequency corresponding to a given position in the focal plane. The zero-order term is the classic diffraction pattern; the first order term is the "pinned speckle" term which modulates the Airy pattern ${ }^{11}$, and the second order term is a combination of the fundamental PSF halo $\left(a^{*} \Phi\right)\left(a^{*} * \Phi^{*}\right)$ with an additional "Strehl" term that serves to remove light from the PSF core. The diffraction pattern and the pinned speckle term dominate at moderate angles for normal AO systems/cameras but can be suppressed by any one of a variety of coronagraphs; a mathematically simple though physically impractical example is apodizing the aperture function with a smoothly-varying $A(\mathbf{x})$ so that $a(\mathbf{k})$ drops rapidly to zero for large $\mathbf{k}$. More practical options include the classic Lyot coronagraph ${ }^{12}$, its modern band-limited descendant ${ }^{13}$ and hybrid apodized Lyot variant ${ }^{14}$, and various binary shaped pupils that produce the effects of apodization $^{15}$. All these options (and many others) suppress the $a(\mathbf{k})$ at large angles. In such a coronagraphic system, once diffraction has been suppressed, the PSF halo term - essentially the power spectrum of the input phase aberration is the primary source of scattered light. (When the second-order term is very small due to high-accuracy wavefront control a fourth-order term becomes significant at some radii ${ }^{16}$, but this generally does not apply in the adaptive optics regime unless there is a large aberration at a single spatial frequency.) Figure 3 illustrates this concept, showing an input phase error and its PSF for two pupils, a hard-edged telescope aperture and an apodized coronagraph.

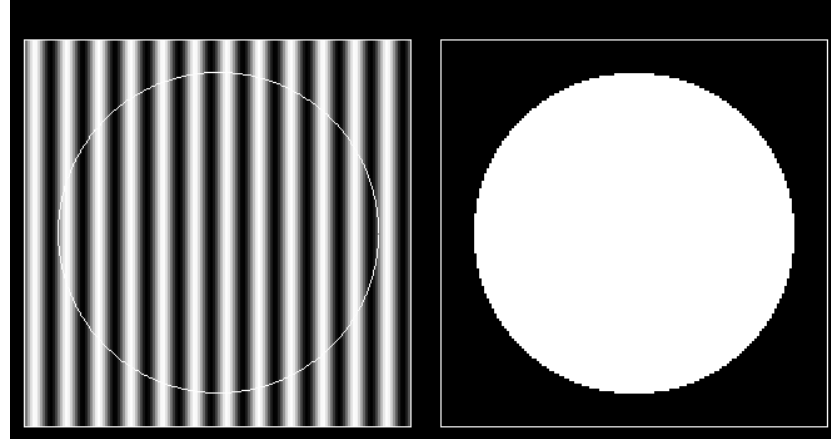

Phose RMS $=1.42 \mathrm{E}-02$
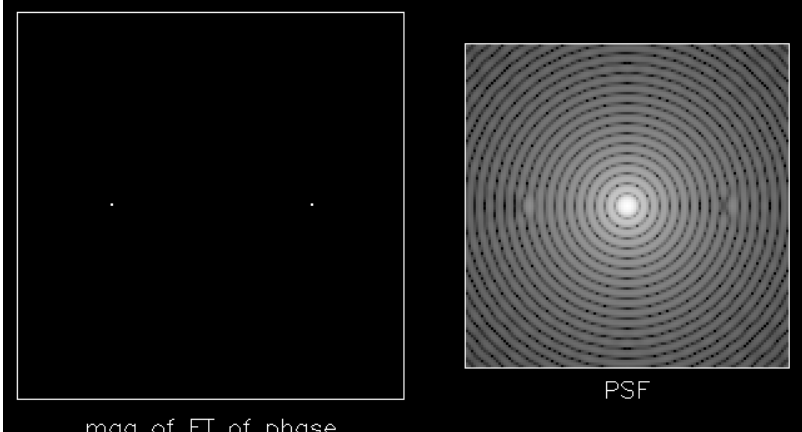

PSF

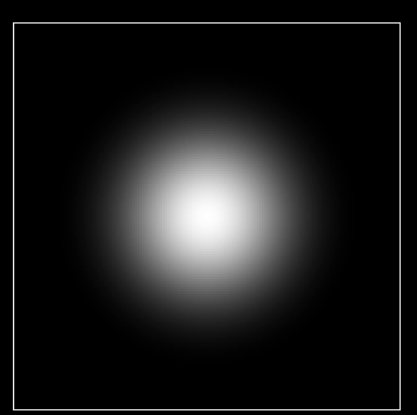

Apod. Pupil

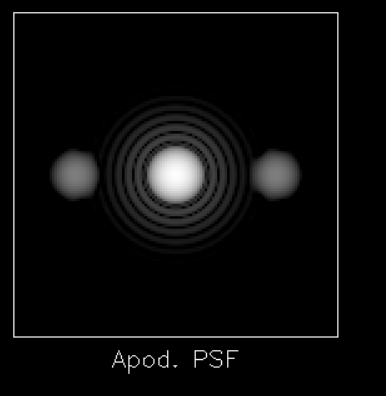

Figure 3: Illustration of the PSF of a coronagraphic and non-coronagraphic system. Upper left: input phase aberration. Upper center: pupil function $A$ for a normal telescope. Lower center: corresponding PSF showing an antisymmertic pair of pinned speckles. Upper right: apodized $A$. Lower right: corresponding PSF showing a symmetric pair of halo speckles.

To detect planets at angular separations $\sim \theta$ we are therefore concerned only with spatial frequencies close to $2 \pi \theta / \lambda$; higher frequency wavefront errors will scatter light to larger angles. A well-behaved continuous deformable mirror 
(DM) with actuator spacing $d$ (projected into the telescope primary mirror) can act as an interpolator. Given perfect knowledge of a desired wavefront $\phi$, the DM can assume a shape $\phi_{\mathrm{dm}}$ such that

$$
\begin{aligned}
\Phi_{D M}(\mathbf{k}) & =\Phi(\mathbf{k}) \text { for }|\mathbf{k}|<4 \pi / d \\
& \approx 0 \quad \text { for }|\mathbf{k}| \geq 4 \pi / d
\end{aligned}
$$

The Fourier transform of the wavefront error $\Phi-\Phi_{\mathrm{dm}}$ will therefore be nearly zero within the controllable range of spatial frequencies and in turn the PSF will have a characteristic square "dark hole" $\lambda / d$ in size (see Figure 6). Segmented deformable mirrors or continuous DMs with broad influence functions may have similar capabilities to reproduce low-frequency signals, but at the cost of injecting significant additional power at higher frequencies.

This requires perfect knowledge of the wavefront and a static phase aberration. Normal AO systems with their finitelysampled wavefront sensors are often subject to aliasing effects which produce spurious low-frequency signals and fill in the dark hole. Techniques such as spatial filtering of the wavefront sensor ${ }^{17}$ can prevent this. Wavefront sensing through focal-plane techniques such as phase retrieval can also produce the necessary information, though not at speeds suitable for ground-based AO. Finite signal-to-noise in the wavefront measurement will produce wavefront errors at the same frequencies the DM can control, as will delays between sensing and correction of the wavefront. The exact spatial frequency of the errors depends on the details of the system and controller ${ }^{18}$, but generally speaking, an ExAO system will produce a PSF similar to Figure 6. To achieve this sort of behavior, the Strehl ratio must be also moderately high $(>0.8)$ to prevent higher-order terms omitted from the above PSF expansion from dominating.

The residual wavefront will have both random components such as atmospheric and measurement errors and quasistatic errors such as miscalibration of non-common-path optical aberrations and chromatic errors caused by the differing wavelengths of the wavefront sensor and science imager. In a long exposure image the PSF components from the random errors will smooth out, but the quasi-static errors will not. These errors must be removed or stabilized at the nanometer level to achieve contrasts $>10^{7} .{ }^{19,20}$

\section{OVERVIEW OF AN EXTREME ADAPTIVE OPTICS SYSTEM}

Figure 4 and Table 1 describe a proposed ExAO system, the Gemini Planet Imager (GPI) intended for the Gemini 8-m telescope(s). The design is from a detailed conceptual design study carried out in 2004-2005 by a collaboration led by the NSF Center for Adaptive Optics ${ }^{21}$.

\begin{tabular}{|l|l|}
\hline AO subsystem & $>48$ actuator diameter MEMS mirror \\
\hline Primary deformable mirror & $\begin{array}{l}d=18 \mathrm{~cm}(N=44 \text { subapertures across primary } \\
\text { mirror) }\end{array}$ \\
\hline Subaperture size & $\begin{array}{l}\text { Spatially-filtered Shack-Hartmann Wave Front } \\
\text { Sensor (SFWFS) }\end{array}$ \\
\hline Wave front sensor type & $128 \times 128$ pixels \\
\hline Wave front sensor CCD & $2500 \mathrm{~Hz}$ \\
\hline Maximum update rate & Optimal Fourier Transform Reconstructor \\
\hline Reconstructor & $I<8$ mag. (goal: $I<9$ mag.) \\
\hline Limting magnitude & $5 \mathrm{~nm}$ RMS surface per powered optic \\
\hline Optics & Apodized-Pupil Lyot Coronagraph (APLC) \\
\hline Surface quality & $\sim 3 \lambda / D$ \\
\hline Coronagraph subsystem & $>60 \%$ \\
\hline Type & Infrared interferometeric wave front sensor \\
\hline Inner working distance & \\
\hline Transmission &
\end{tabular}




\begin{tabular}{|l|l|}
\hline Wavelength range & $1-2.4 \mu \mathrm{m}$ \\
\hline Wave front measurement precision & $1 \mathrm{~nm}$ RMS in controlled frequency range \\
\hline Science Instrument & Lenslet-based integral field spectrograph \\
\hline Type & $0.014 \times 0.014$ arcseconds \\
\hline Lenslet size & $3.6 \times 3.6$ arcseconds \\
\hline Field of view & $\lambda / \Delta \lambda \sim 40$ \\
\hline Spectral resolution & Single $20 \%$ band within $1-2.4 \mu \mathrm{m}$ range \\
\hline Spectral coverage & HAWAII-II RG HgCdTe 2048x2048 pixel IR array \\
\hline Detector
\end{tabular}

Table 1: Summary of properties of the Gemini Planet Imager AO planet detection system

EXAOC SYSTEM OVERVIEW

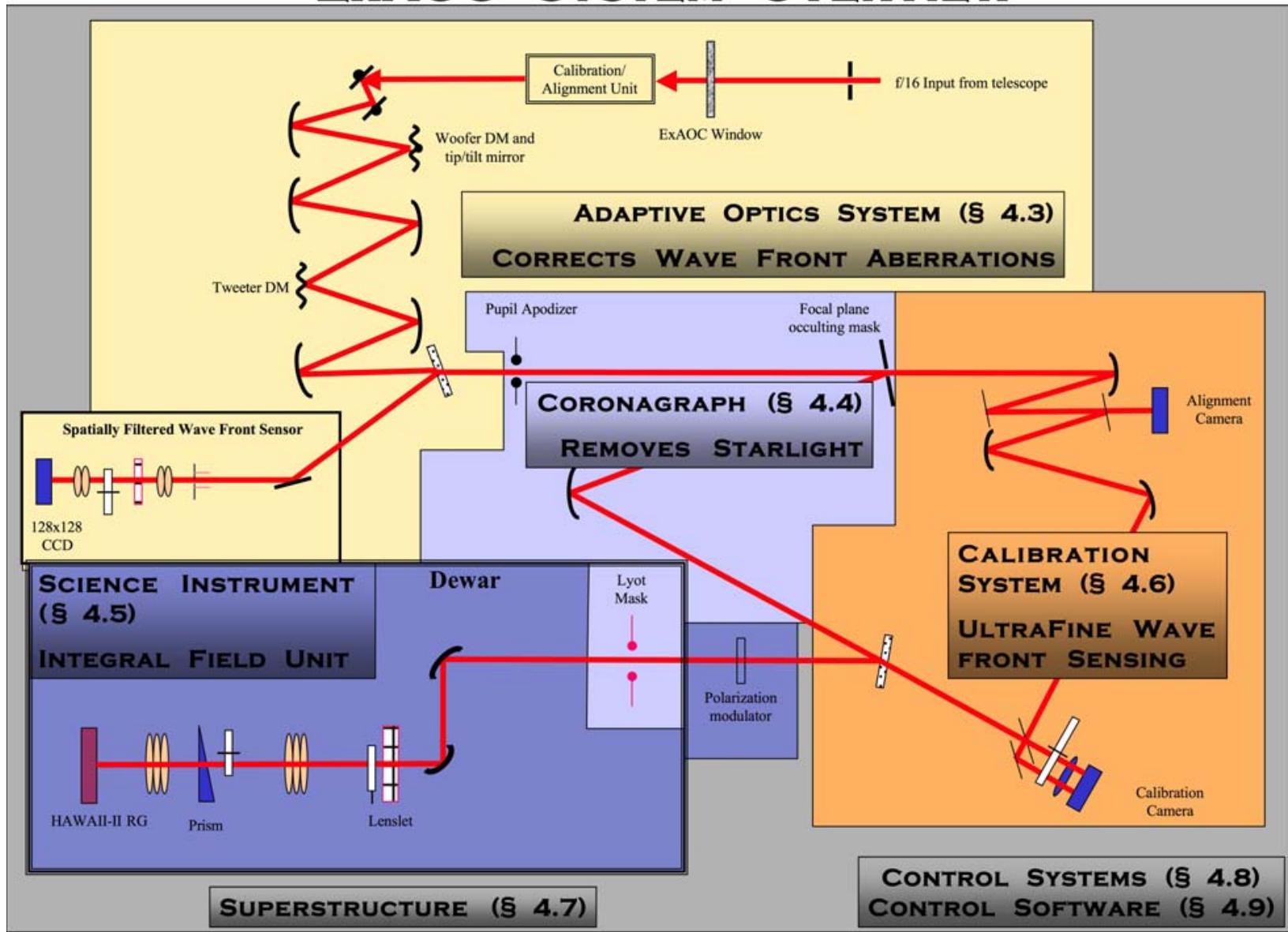

Figure 4: Schematic layout of the Gemini Planet Imager (previously known as "ExAOC") instrument. Section numbers refer to chapters in the GPI/ExAOC conceptual design study ${ }^{21}$.

Key features of the design:

- A typical ExAO system intended to discover planets at $\theta<0.9$ arcsecond at a wavelength of $1.6 \mu \mathrm{m}$ therefore requires $d \sim 18 \mathrm{~cm}$. For an 8 -m telescope, this corresponds to 44 subapertures across the diameter of the primary mirror; allowing for a 2-actuator band of slaved guard actuators, this is $\sim 1800$ controlled deformable mirror actuators. While such a high actuator count is barely feasible with conventional piezo-DM technology, it is much more practical with MEMS-based devices.

- Fully correcting the tilt-removed atmosphere on a $8-\mathrm{m}$ telescope requires $\sim 8 \mu \mathrm{m}$ of physical stroke. Since current MEMS-based continuous deformable mirrors do not have this range, GPI operates with two deformable 
mirrors; a high-stroke low-actuator-count "woofer" and a MEMS-based "tweeter". Low-order wavefront components are offloaded to the woofer. Table 2 shows the 1-sigma wavefront residuals after woofer correction for a variety of woofer choices. Setting the tweeter stroke to 5-6 times the one-sigma level will cause saturation to be acceptably rare.

- The wavefront sensor is a spatially-filtered Shack-Hartmann sensor ${ }^{17}$ operating at 0.7-0.9 $\mu \mathrm{m}$.

- To control $\sim 1800$ actuators at $2500 \mathrm{~Hz}$ rates with current computer technology, GPI will employ an efficient Fourier Transform wavefront reconstructor running on off-the-shelf hardware ${ }^{22}$.

- All optics must be of very high quality ( $<5 \mathrm{~nm}$ RMS) to minimize the effects of static wavefront errors. This favors a compact MEMS-based device with correspondingly small internal optics.

- The apodized-pupil Lyot coronagraph ${ }^{14}$ suppresses residual diffraction.

- A precision infrared wavefront sensor, integrated with the coronagraph, measures the time-averaged science wavefront to correct for quasi-static and chromatic aberrations.

- The science instrument is a integral field spectrograph, similar to OSIRIS ${ }^{23}$ but with lower spectral resolution and a much larger field of view. This allows rejection of PSF artifacts through their chromatic behavior ${ }^{24,25}$ while also producing low-resolution spectra of any planets that are discovered.

\begin{tabular}{|l|l|l|}
\hline \multicolumn{1}{|c|}{ Woofer DM } & Full pupil residuals $(\boldsymbol{\mu m})$ & Inner 90\% residuals $(\boldsymbol{\mu m})$ \\
\hline Tip/tilt only & 1.30 & 1.11 \\
\hline 177 actuator PMN mirror & 0.29 & 0.19 \\
\hline 104 actuator bimorph mirror & 0.25 & 0.25 \\
\hline 36 actuator bimorph mirror & 0.36 & 0.33 \\
\hline 19 actuator bimorph mirror & 0.49 & 0.40 \\
\hline
\end{tabular}

Table 2: Wavefront error left to the MEMS DM after woofer correction. Results are 1-sigma standard deviation for $r_{0}=15 \mathrm{~cm} @ 0.5 \mu \mathrm{m}$. They scale as $r_{0}^{6 / 5}$

\begin{tabular}{|l|c|c|}
\hline \multicolumn{1}{|c|}{ Property } & Requirement & Notes \\
\hline Pixel count & $4096(64 \mathrm{x} 64)$ & $\begin{array}{c}\text { GPI will use } 48 \text {-actuator } \\
\text { circle }\end{array}$ \\
\hline Pitch & 300 to 400 microns & $\begin{array}{c}\text { Depends on properties of } \\
\text { woofer mirror }\end{array}$ \\
\hline $\begin{array}{l}\text { Stroke (overall peak-to- } \\
\text { valley) }\end{array}$ & $2.4 \mu \mathrm{m}$ (surface) & \\
\hline $\begin{array}{l}\text { Stroke at highest controllable } \\
\text { spatial frequency }\end{array}$ & $1 \mu \mathrm{m}$ (surface) & \\
\hline Fill Factor & $99 \%$ & \\
\hline Aperture size & 19.2 to $25.6 \mathrm{~mm}$ & \\
\hline Pixel surface finish (RMS) & $<10 \mathrm{~nm}$ (goal: $<3 \mathrm{~nm})$ & \\
\hline $\begin{array}{l}\text { Pixel surface finish (peak to } \\
\text { valley) }\end{array}$ & $<30 \mathrm{~nm}$ (goal: $<9 \mathrm{~nm})$ & \\
\hline $\begin{array}{l}\text { Wavefront error after } \\
\text { flattening within controlled } \\
\text { spatial frequency range } \\
\text { (RMS) }\end{array}$ & $<1 \mathrm{~nm}$ & $\begin{array}{c}\text { MEMS mirrors in air will be } \\
\text { damped and may not have } \\
\text { resonances }\end{array}$ \\
\hline Bandwidth (1 dB) & $>250 \mathrm{~Hz}$ & \\
\hline First resonance & $>2500 \mathrm{~Hz}$ & \\
\hline $\begin{array}{l}\text { Neighboring actuator } \\
\text { differential stroke }\end{array}$ & $>1 \mathrm{um}$ & \\
\hline Actuator yield & $>99.8 \%$ & \\
\hline Clear aperture & $\begin{array}{l}\text { Fully-functional } 48 \text {-actuator } \\
\text { circle at least } 2 \mathrm{actuators} \text { away } \\
\text { from edge }\end{array}$ & \\
\hline
\end{tabular}




\begin{tabular}{|l|c|c|}
\hline \multicolumn{1}{|c|}{ Property } & Requirement & Notes \\
\hline Reflective surface & Gold-coated continuous facesheet & \\
\hline $\begin{array}{l}\text { Uniformity of surface } \\
\text { reflectivity }\end{array}$ & $+/-1 \%$ RMS & \\
\hline Maximum drive voltage & $300 \mathrm{~V}$ & \\
\hline Operating temperature & $-30^{\circ}$ to $+30^{\circ} \mathrm{C}$ & \\
\hline
\end{tabular}

Table 3: MEMS mirror requirements for GPI

Table 3 lists the properties required of the MEMS deformable mirror.

- ExAO imaging is highly sensitive to failed actuators, which will cause wavefront errors at all spatial frequencies and scatter light within the dark hole. It may be possible to mask an isolated actuator with the coronagraph pupil mask, but since this generally requires masking an area of radius $10-20 \%$ of the pupil diameter around each failed actuator, only a few failed actuators can be corrected this way. One approach to increasing the probability of this is to manufacture 64x64 actuator MEMS devices and then attempt to find a 48 -actuator clear circle; if a $64 \times 64$ device is not significantly more expensive than a $48 \times 48$ device this will substantially increase the probability of finding a suitable mirror. Monte Carlo simulations (Figure 5) show that with a $99.8 \%$ actuator yield, the probability of finding a suitable region on a $48 \times 48$ device is $\sim 20 \%$, sufficiently high that a single fabrication run will be almost guaranteed to yield a suitable device.

- As discussed in Section 2, high-frequency aberrations primarily scatter light to large angles. However, if the aberrations are large $(>10 \mathrm{~nm})$ and concentrated at a few spatial frequencies they can also scatter light into the dark hole region through higher-order terms in the PSF expansion ${ }^{10,16}$; the most significant term has the form $\left[\left(a^{*} \Phi\right)\left(a^{*} * \Phi^{*}\right)\right] *\left[\left(a^{*} \Phi\right)\left(a^{*} * \Phi^{*}\right)\right]$, the convolution of the wavefront power spectrum with itself. If the wavefront errors are concentrated at a single spatial frequency, e.g. $\mathbf{k}=2 \pi / d$ for actuator print-through, this will produce copies of the main PSF centered at offsets of $\lambda / \mathrm{d}$, which can in turn lead to quasi-static artifacts in the region of interest.

- For similar reasons, continuous mirrors are preferred over segmented devices. Simulations show that a segmented mirror with individual tip/tilt/piston control can give acceptable performance, but requires the actual segments to be quite flat $(<10 \mathrm{~nm}$ RMS $)$ to minimize phase discontinuities at the edges. Gaps between the segments will also scatter light.

- ExAO systems operate at very high update rates $(>2000 \mathrm{~Hz})$, and the MEMS must respond at these rates.

- Continuous or especially membrane MEMS mirrors often have relatively broad influence functions, which can reduce stroke at the highest frequencies. With low spatial frequencies corrected by the woofer mirror, the highfrequency requirements often set overall stroke.

- MEMS flattening at the sub-nanometer level has been demonstrated in the laboratory. ${ }^{26}$

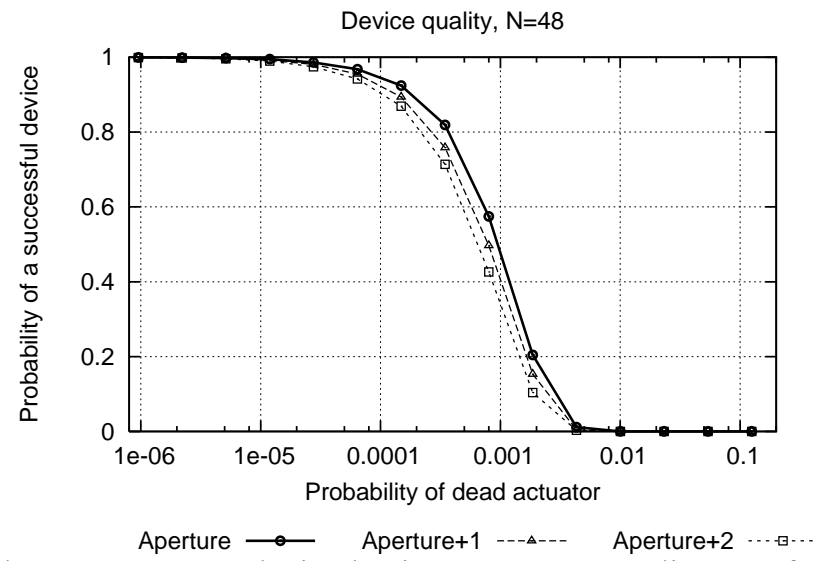

Figure 5: Probability of a single 64x64 MEMS device having a 48-actuator-diameter functional circle, where 0,1 or 2 slave actuators surrounding the circle are required, as a function of actuator failure probability.

Figure 6 shows a simulated short-exposure image. The ultimate sensitivity metric will be the number of planets detected, which depends on the (unknown) properties of planets in orbits $>5 \mathrm{AU}$ in the solar neighborhood. Figure 6 
also shows the results of a Monte Carlo simulation of the observation of $I<8$ mag. stars, based on an extrapolation of planet properties from the radial velocity samples. If planets continue to be plentiful at $>5 \mathrm{AU}$ separations, GPI can expect to discover large number of such planets.
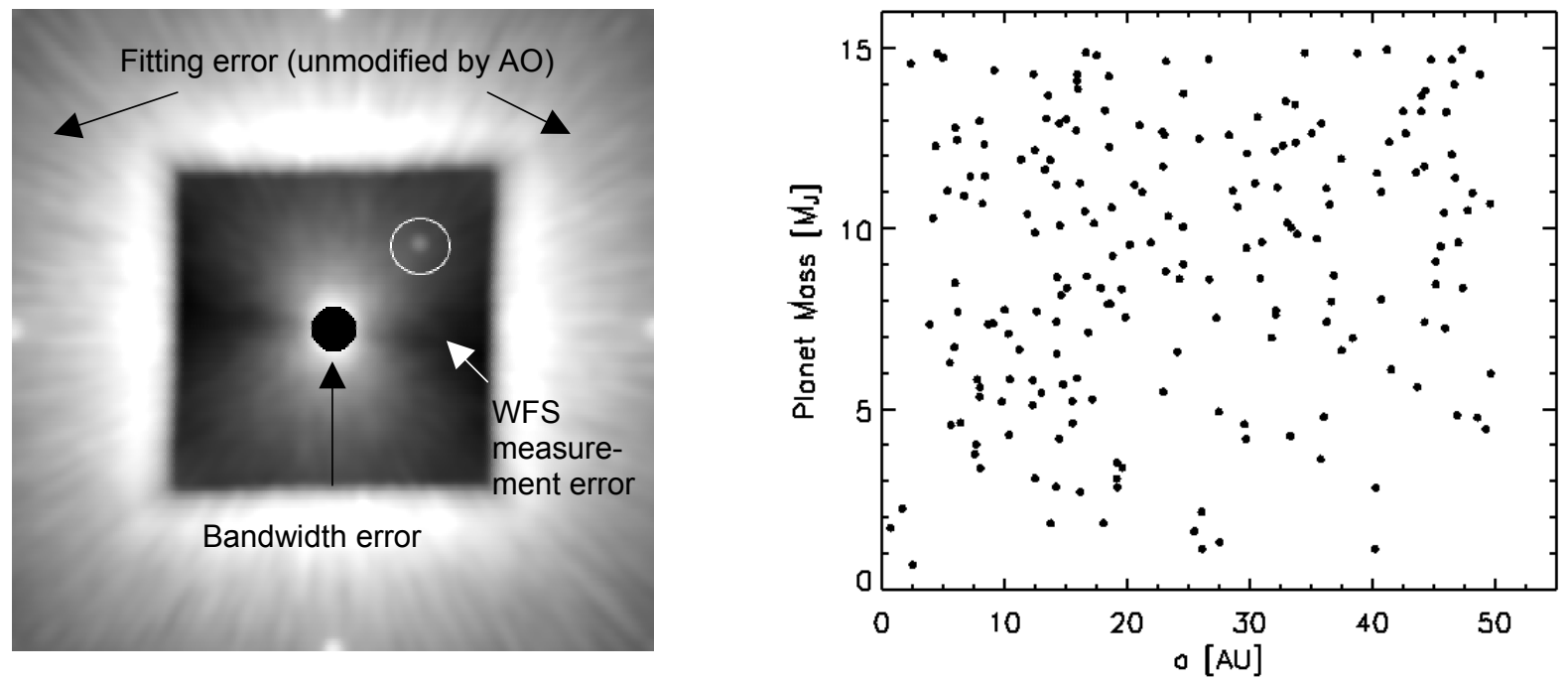

Figure 6: Left: Simulated ExAO system PSF. Different regions of the PSF are dominated by speckles caused by different error sources, as indicated. Right: Planets discovered in a Monte Carlo simulation of a GPI survey of $I<8$ mag stars in the solar neighborhood, assuming a planet distribution extrapolated from current radial velocity surveys, the AO system described above, and a median $\mathrm{r}_{0}=14 \mathrm{~cm}$. X axis is orbital semi-major-axis (AU), Y axis is planet mass in Jupiter masses.

\section{CONCLUSIONS}

Although challenging, direct detection of extrasolar planets appears feasible with current technology. This will require continuous-facesheet deformable mirrors with $\sim 48$ actuator diameter and essentially no defective actuators. MEMSbased deformable mirrors will allow a compact system - crucial to keeping instrument cost manageable, especially given the high quality internal optics required. Such MEMS require larger devices than are currently available, and improvements to actuator yield, but appear practical with current technology. The Gemini Planet Imager has been designed around a MEMS deformable mirror and is expected to be deployed on the telescope by 2010.

\section{ACKNOWLEDGEMENTS}

This research was performed under the auspices of the U.S. Department of Energy by the University of California, Lawrence Livermore National Laboratory under Contract W-7405-ENG-48, and also supported in part by the National Science Foundation Science and Technology Center for Adaptive Optics, managed by the University of California at Santa Cruz under cooperative agreement No. AST - 9876783. The Gemini Observatory is operated by the Association of Universities for Research in Astronomy, Inc., under a cooperative agreement with the NSF on behalf of the Gemini partnership: the National Science Foundation (United States), the Particle Physics and Astronomy Research Council (United Kingdom), the National Research Council (Canada), CONICYT (Chile), the Australian Research Council (Australia), CNPq (Brazil), and CONICET (Argentina).

\section{REFERENCES}

\footnotetext{
${ }^{1}$ See http://vo.obspm.fr/exoplanetes/encyclo/catalog.php or http://exoplanets.org for a current list.
} 
${ }^{2}$ Lin, D. N. C. \& Ida, S. 1997, Ap.J., 477, 781

${ }^{3}$ Rasio, F. A. \& Ford, E. B. 1996, Science, 274, 954

${ }^{4}$ Boss, A.P. 2002, Ap..J, 576, 462

${ }^{5}$ Marcy, G. W., Butler, R. P., Fischer, D. A., Vogt, S. S. 2003, ASP Conference series, 294, 1

${ }^{6}$ Chauvin, G., et al. 2004 A\&A 425, L29

${ }^{7}$ Burrows, A. J., et al. 1997, Ap.J., 491, 856

${ }^{8}$ Max, C.E., et al. 1997 Science, 277, 1649

${ }^{9}$ Sivaramakrishnan, A., Lloyd, J. P., Hodge, P. E., and Macintosh, B. A. 2002, Ap.J. 581, L59

${ }^{10}$ Perrin, M., et al. 2003 Ap.J. 596, 702

${ }^{11}$ Bloemhof, E., et al. 2001 Ap.J. 558, L71

${ }^{12}$ Lyot, B. 1939, MNRAS, 99, 580

${ }^{13}$ Kuchner, M. J. \& Traub, W. A. 2002, ApJ, 570, 900

${ }^{14}$ Soummer, R. 2005, ApJ, 618, L161

${ }^{15}$ Kasdin, N. J., Vanderbei, R. J., Spergel, D. N. \& Littman, M. G. 2003, ApJ, 582, 1147

${ }^{16}$ Give'on, A., et al 2006 JOSA A in press

${ }^{17}$ Poyneer, L, and Macintosh, B., 2004 JOSA A 21, 810

${ }^{18}$ Rigaut, F., Veran, J.-P., and Lai, O., 1998 Proc. SPIE 3353, 1038

${ }^{19}$ Wallace, J.K., et al. 2004 Proc. SPIE 5490, 370

${ }^{20}$ Macintosh, B., et al. 2004 Proc. SPIE 5490, 359

${ }^{21}$ Macintosh, B., et al 2005 Extreme Adaptive Optics Coronagraph conceptual design study, LLNL

${ }^{22}$ Poyneer, L. A., Gavel, D.T., and Brase, J.M., 2002 JOSA A 19, 2100

${ }^{23}$ Larkin, J., et al 2005 Proc. SPIE 4841, 1600

${ }^{24}$ Marois, C., et al. 2000 PASP 112, 91

${ }^{25}$ Sparks, W.B. \& Ford, H.C. 2002, ApJ, 578, 543

${ }^{26}$ Evans, J., et al. 2006 Proc. SPIE this volume 